\title{
APPROXIMATION THEOREMS FOR NASH MAPPINGS AND NASH MANIFOLDS
}

\author{
BY
}

\author{
MASAHIRO SHIOTA
}

\author{
Dedicated to Professor Nobuo Shimada on his 60 th birthday
}

\begin{abstract}
Let $0 \leqslant r<\infty$. A $C^{r}$ Nash function on $\mathbf{R}^{n}$ is a $C^{r}$ function whose graph is semialgebraic. It is shown that a $C^{r}$ Nash function is approximated by a $C^{\omega} \mathrm{Nash}$ one in a strong topology defined in the same way as the usual topology on the space $\mathscr{S}$ of rapidly decreasing $C^{\infty}$ functions. A $C^{r}$ Nash manifold in $\mathbf{R}^{n}$ is a semialgebraic $C^{r}$ manifold. We also prove that a $C^{r}$ Nash manifold for $r \geqslant 1$ is approximated by a $C^{\omega}$ Nash manifold, from which we can classify all $C^{r}$ Nash manifolds by $C^{r}$ Nash diffeomorphisms.
\end{abstract}

1. Introduction. Let $r=0, \ldots, \infty$ or $\omega$. A submanifold of $\mathbf{R}^{n}$ is called a $C^{r}$ Nash manifold if it is semialgebraic and of class $C^{r}$. A $C^{r}$ map from one $C^{r}$ Nash manifold to another is called a $C^{r}$ Nash map if the graph is semialgebraic. We define similarly a $C^{r}$ Nash vector field. For a $C^{r}$ Nash manifold $M$, let $N^{r}(M)$ denote the ring of all $C^{r}$ Nash functions on $M$. As a $C^{\infty}$ Nash manifold and a $C^{\infty}$ Nash map are automatically of class $C^{\omega}$ (Proposition 3.11, [14]), we assume $r \neq \infty$.

Our purpose is to approximate a $C^{r}$ Nash manifold and a $C^{r}$ Nash map between $C^{\omega}$ Nash manifolds by $C^{\omega}$ Nash ones. If the manifolds are compact, the problem is easy (see [7 and 8]). In fact a $C^{r}$ map between $C^{\omega}$ compact Nash manifolds is approximated by a $C^{\omega}$ Nash map in the $C^{r}$ topology, and for a compact $C^{r}$ Nash manifold $M \subset \mathbf{R}^{n}(r \geqslant 1)$ there exists a $C^{r}$ Nash imbedding $\tau$ of $M$ in $\mathbf{R}^{n}$ arbitrarily close to the identity in the $C^{r}$ topology such that $\tau(M)$ is a $C^{\omega}$ Nash manifold.

When we consider the noncompact case, the compact-open or uniform $C^{r}$ topology on $N^{r}(M)$ is too weak to apply approximation theorems (indeed, for example, polynomial approximation works only in the compact-open $C^{r}$ topology and is not useful to investigate noncompact manifolds). So we use a stronger topology defined as follows. Let $f_{k} \in N^{r}(M), k=1,2, \ldots$. We define $f_{k} \rightarrow 0$ when $v_{1} \cdots v_{r^{\prime}} f$ uniformly converges to 0 for any $C^{r}$ Nash vector fields $v_{1}, \ldots, v_{r^{\prime}}$ with $\infty>r^{\prime} \leqslant r$. When $M=\mathbf{R}^{n}$ and $r=\infty$ this coincides with the usual topology on $\mathscr{S}$ (the space of rapidly decreasing $C^{\infty}$ functions [3]). Namely $f_{k} \rightarrow 0$ if and only if $x^{\alpha} D^{\beta} f_{k}(x)$ uniformly converges to 0 for any multi-indices $\alpha$ and $\beta$. We remark that $N^{r}(M)$ in this topology is not a linear topological space since af does not converge

Received by the editors December 3, 1984 and, in revised form, March 4, 1985.

1980 Mathematics Subject Classification. Primary 58A07; Secondary 14G30.

Key words and phrases. Nash manifold, real algebraic geometry, semialgebraic set.

(c1986 American Mathematical Society $0002-9947 / 86 \$ 1.00+\$ .25$ per page 
to 0 as $a \in \mathbf{R} \rightarrow 0$ unless the support of $f$ is compact. In this paper we always treat this topology and we call it simply the $C^{r}$ topology.

THEOREM 1. Let $M_{1}$ and $M_{2}$ be $C^{\omega}$ Nash manifolds and $f: M_{1} \rightarrow M_{2}$ a $C^{r}$ Nash map. Then $f$ can be approximated by a $C^{\omega}$ Nash map in the $C^{r}$ topology. Moreover assume the restriction of $f$ to a given compact $C^{\omega}$ Nash submanifold $M_{3}$ of $M_{1}$ to be of class $C^{\omega}$. Then we can approximate f fixing on $M_{3}$.

Corollary 2. Let $U_{1}$ and $U_{2}$ be open semialgebraic sets in $\mathbf{R}^{n}$ with $\bar{U}_{1} \subset U_{2}$ and let $f$ be a $C^{\omega}$ Nash function on $U_{2}$. Then there exists a $C^{\omega}$ Nash function $g$ on $\mathbf{R}^{n}$ such that $\left.g\right|_{U_{1}}$ is an approximation of $\left.f\right|_{U_{1}}$ in the $C^{\omega}$ topology.

Efroymson [2] stated Corollary 2 in the $C^{0}$ topology and the author is greatly inspired by that paper. To prove Theorem 1 we use a partition of unity by $C^{r} \mathrm{Nash}$ functions, whose existence is shown in $\$ 2$ and it clarifies a difference between Nash functions and polynomials or rational functions.

THEOREM 3. Let $M \subset \mathbf{R}^{n}$ be a $C^{r}$ Nash manifold with $1 \leqslant r<\infty$. Then there exists a $C^{r}$ Nash imbedding $\tau$ of $M$ in $\mathbf{R}^{n}$ arbitrarily close to the identity in the $C^{r}$ topology such that $\tau(M)$ is a $C^{\omega}$ Nash manifold. Moreover for any compact $C^{\omega}$ Nash manifold $M_{1}$ contained in $M$ we can choose $\tau$ so that $\left.\tau\right|_{M_{1}}=$ ident. Another additional property is: Given a $C^{r}$ Nash manifold $M_{2}$ contained in $M$ but not necessarily closed in $M$, we can require that $\tau\left(M_{2}\right)$ is of class $C^{\omega}$ Nash.

If $r=0$, then $\tau(M)$ becomes a PL manifold [12] and the interior of a compact PL manifold possibly with boundary is of class $C^{0} \mathrm{Nash}$.

Let $N_{1}, N_{2}$ and $N_{3}$ be the $C^{r}$ Nash diffeomorphism classes of all compact $C^{r}$ Nash manifolds possibly with boundary, the $C^{r}$ diffeomorphism classes of the same ones and the $C^{r}$ Nash diffeomorphism classes of all $C^{r}$ Nash manifolds respectively. Then we have natural maps $i_{1}: N_{1} \rightarrow N_{2}$ and $i_{2}: N_{1} \rightarrow N_{3}$ defined by $i_{2}(M)=M-\partial M$.

COROllary 4. $i_{1}$ and $i_{2}$ are bijective.

This in the case of $r=\omega$ or $r=0$ is proved in $[9,12]$, respectively, from which, along with Theorem 3 , the case $0<r<\omega$ follows immediately.

$\S 3$ proves Theorem 1 and Corollary 2, and applying Theorem 1 we study $C^{r} \mathrm{Nash}$ manifold structure in $\S 4$. In $\S 4$ we also show the unique existence of $C^{r} \mathrm{Nash}$ vector bundle structure on a $C^{0}$ vector bundle over a $C^{r}$ Nash manifold, which we see without any trouble in the case of compact base space.

2. Partition of unity. Let $X \subset \mathbf{R}^{n}$ be an algebraic set. We shall construct a $C^{\omega} \mathrm{Nash}$ function on $\mathbf{R}^{n}$ which is an approximation of 0 outside a small semialgebraic neighborhood of $X$ and of 1 in another one. The function is required to hold a useful well-known property of a $C^{\infty}$ partition of unity (Proposition 2.5). Let $f \in N^{r}\left(\mathbf{R}^{n}\right.$ ) and $e(x)=1 /\left(C+|x|^{2 k}\right)$, where $C$ is a positive number, $k$ is a positive integer and $|x|^{2}=x_{1}^{2}+\cdots+x_{n}^{2}$. We write $e$ as $e_{C, k}$ when we need to emphasize $C$ and $k$. Let $U \subset \mathbf{R}^{n}$ be an open semialgebraic neighborhood of $f^{-1}(0)$. Put

$$
V_{1}=\{x \notin U: f(x)>0\}, \quad V_{2}=\{x \notin U: f(x)<0\} .
$$


LEMMA 2.1.

$$
F=\left(\left(f^{2}+e\right)^{1 / 2}+f\right) / 2 \rightarrow \begin{cases}0 & \text { on } V_{2}, \\ f & \text { on } V_{1},\end{cases}
$$

in the $C^{r}$ topology as $C$ and $k \rightarrow \infty$ satisfying $k^{2 k} \leqslant C$.

Proof. We can assume $r<\infty$. As the problem is to prove $\left(f^{2}+e\right)^{1 / 2} \rightarrow|f|$ on $V_{1} \cup V_{2}$, it is sufficient to consider the convergence on $V_{1}$. We prove it by induction on $r$.

Case $r=0$. Let $\varepsilon$ be a Nash function of the same form as the above $e$. Put

$$
\psi(t)=\inf \left\{f(x):|x|=t \text { and } x \in V_{1}\right\} .
$$

Then by the Tarski-Seidenberg Theorem, $\psi$ is a positive upper semicontinuous semialgebraic function on the closed semialgebraic set $W=\left\{|x|: x \in V_{1}\right\}$ (here a semialgebraic function means that the graph is semialgebraic). Hence it follows from Lojasiewicz' inequality [5] and the stereographic projection that there exist $C_{1}$, $k_{1}>1$ such that for any $C \geqslant C_{1}$ and $k \geqslant k_{1}$

$$
\varepsilon(t) \psi(t) \geqslant 1 /\left(C+t^{2 k}\right) \text { for } t \in W
$$

where $\varepsilon(t)$ is defined so that $\varepsilon(|x|)=\varepsilon(x)$, in other words

$$
\varepsilon(x) f(x) \geqslant e_{C, k}(x) \text { for } x \in V_{1} \text {. }
$$

Hence we have

$$
0<\left(f^{2}+e\right)^{1 / 2}-f=e /\left(\left(f^{2}+e\right)^{1 / 2}+f\right)<e / 2 f \leqslant \varepsilon / 2 \text { on } V_{1},
$$

which proves Case $r=0$.

Assume $\left(f^{2}+e\right)^{1 / 2} \rightarrow f$ in the $C^{r-1}$ topology, to be precise, for any $\varepsilon$ as above there exist $C_{2}, k_{2} \geqslant 1$ such that for any $C \geqslant C_{2}, k \geqslant k_{2}$ with $k^{2 k} \leqslant C$ and a multi-index $\alpha$ with $|\alpha| \leqslant r-1$

$$
\left|D^{\alpha}\left(f^{2}+e_{C, k}\right)^{1 / 2}-D^{\alpha} f\right| \leqslant \varepsilon \text { on } V_{1} .
$$

We need to prove this inequality for all $\alpha$ with $|\alpha|=r$. Let $\alpha$ be such a one. Obviously

$$
\begin{aligned}
D^{\alpha} e= & D^{\alpha}\left\{\left(\left(f^{2}+e\right)^{1 / 2}-f\right)\left(\left(f^{2}+e\right)^{1 / 2}+f\right)\right\} \\
= & \left\{D^{\alpha}\left(\left(f^{2}+e\right)^{1 / 2}-f\right)\right\}\left\{\left(f^{2}+e\right)^{1 / 2}+f\right\} \\
& +\sum_{\substack{\beta+\gamma=\alpha \\
\gamma \neq 0}}\left\{D^{\beta}\left(\left(f^{2}+e\right)^{1 / 2}-f\right)\right\}\left\{D^{\gamma}\left(\left(f^{2}+e\right)^{1 / 2}+f\right)\right\}
\end{aligned}
$$

and we have constants $d_{0}, \ldots, d_{r-1}$ which depend on $r$ but not on $C$ nor $k$ such that

$$
\left|D^{\alpha} e(x)\right| \leqslant \sum_{0 \leqslant i<r} d_{i} k^{r}|x|^{(r-i)(2 k-1)-i} /\left(C+|x|^{2 k}\right)^{r-i+1} \text {. }
$$

Easy calculations show

$$
k^{r}|x|^{(r-i)(2 k-1)-i} /\left(C+|x|^{2 k}\right)^{r-i} \leqslant 1
$$


by $k \leqslant k^{2 k} \leqslant C$. Hence we have

$$
\left|D^{\alpha} e\right| \leqslant \sum_{i=0}^{r-1} d_{i} e,
$$

which, together with the induction hypothesis, implies

$$
\begin{aligned}
& \left|D^{\alpha}\left(\left(f^{2}+e\right)^{1 / 2}-f\right)\right| \\
& \quad \leqslant\left\{d e+\varepsilon_{1} \sum_{0<\gamma \leqslant \alpha}\left|D^{\alpha}\left(\left(f^{2}+e\right)^{1 / 2}+f\right)\right|\right\} /\left(\left(f^{2}+e\right)^{1 / 2}+f\right) \text { on } V_{1}
\end{aligned}
$$

for any $\varepsilon_{1}$ as $\varepsilon$ and sufficiently small $e$ with $k^{2 k} \leqslant C$, where $d=\sum_{i=0}^{r-1} d_{i}$. Therefore by the same argument as the case $r=0$, choosing small $\varepsilon_{1}$ we obtain $C_{3}, k_{3} \geqslant 1$ such that for any $C \geqslant C_{3}$ and $k \geqslant k_{3}$ with $k^{2 k} \leqslant C$

$$
\left|D^{\alpha}\left(\left(f^{2}+e\right)^{1 / 2}-f\right)\right| \leqslant \varepsilon \text { on } V_{1},
$$

which proves the lemma.

Definition. We call the argument for $r=0$ in the above proof Argument 2.1.

Let $\infty>r^{\prime} \leqslant r$ and let $\varphi$ be a polynomial on $\mathbf{R}$ such that $\varphi(0)=\cdots=\varphi^{\left(r^{\prime}\right)}(0)=$ 0 . Then

$$
\varphi\{(|f|+f) / 2\}
$$

is a $C^{r^{\prime}}$ Nash function $r^{\prime}$-flat at $f^{-1}(0)$ (i.e. $D^{\alpha} \varphi\{(|f|+f) / 2\} \equiv 0$ on $f^{-1}(0)$ when $\left.|\alpha| \leqslant r^{\prime}\right)$.

Lemma 2.2. $\varphi(F) \rightarrow \varphi\{(|f|+f) / 2\}$ in the $C^{r^{\prime}}$ topology as $C$ and $k \rightarrow \infty$ satisfying $k^{2 k} \leqslant C$, where $F$ is defined in Lemma 2.1.

Proof. Put $f_{1}=(|f|+f) / 2$. Let $\alpha$ be a multi-index with $|\alpha| \leqslant r^{\prime}$. Obviously if $|\alpha|>0$

$$
\begin{gathered}
D^{\alpha} \varphi(F)=\varphi^{\prime}(F) D^{\alpha} F+\varphi^{\prime \prime}(F) \sum_{\substack{\beta+\gamma=\alpha \\
\beta, \gamma \neq 0}} D^{\beta} F D^{\gamma} F+\cdots, \\
D^{\alpha} \varphi\left(f_{1}\right)=\left\{\begin{array}{l}
0 \text { on } f^{-1}(0), \\
\varphi^{\prime}\left(f_{1}\right) D^{\alpha} f_{1}+\varphi^{\prime \prime}\left(f_{1}\right) \sum_{\substack{\beta+\gamma=\alpha \\
\beta, \gamma \neq 0}} D^{\beta} f_{1} D^{\gamma} f_{1}+\cdots
\end{array} \text { outside } f^{-1}(0) .\right.
\end{gathered}
$$

Hence, for any open semialgebraic neighborhood $U$ of $f^{-1}(0)$, the convergence $D^{\alpha} \varphi(F) \rightarrow D^{\alpha} \varphi\left(f_{1}\right)$ on $\mathbf{R}^{n}-U$ in the $C^{0}$ topology follows from Lemma 2.1. So it suffices to prove the following.

Let $\alpha_{1}, \ldots, \alpha_{l}>0$ be multi-indices with $\left|\alpha_{1}\right|+\cdots+\left|\alpha_{l}\right|=r^{\prime \prime} \leqslant r^{\prime}$, and let $\varepsilon$ be a Nash function of the same form as $e$. Then there exist $C_{1}, k_{1} \geqslant 1$ and an open semialgebraic neighborhood $U$ of $f^{-1}(0)$ such that for any $C \geqslant C_{1}$ and $k \geqslant k_{1}$ with $k^{2 k}<C$

$$
\begin{array}{ll}
\left|\varphi^{(l)}(F) D^{\alpha_{1}} F \cdots D^{\alpha_{l}} F\right|<\varepsilon & \text { on } U, \\
\left|\varphi^{(l)}\left(f_{1}\right) D^{\alpha_{1}} f_{1} \cdots D^{\alpha_{l}} f_{1}\right|<\varepsilon & \text { on } U-f^{-1}(0) .
\end{array}
$$


As the existence of $U$ which satisfies the second inequality is trivial, we consider only the first one. By assumption, $\varphi^{(l)}(F)=F^{r^{\prime \prime}-l+1} \psi(F)$ for some polynomial $\psi$. Hence, by Argument 2.1 it suffices to prove

$$
\left|F^{r^{\prime \prime}-l+1} D^{\alpha_{1}} F \cdots D^{\alpha_{l}} F\right|<\varepsilon \text { on } U,
$$

which is equivalent to

$$
\left|f_{2}^{r^{\prime \prime}-l+1} D^{\alpha_{1}}\left(f_{2}+f\right) \cdots D^{\alpha_{l}}\left(f_{2}+f\right)\right|<\varepsilon \text { on } U,
$$

where $f_{2}=\left(f^{2}+e\right)^{1 / 2}$, because of $f_{2} / 2<|F|<f_{2}$. Moreover, by induction on $r^{\prime \prime}$ (see below) and Argument 2.1, this inequality is reduced to

$$
\left|f_{2}^{r^{\prime \prime}-l+1} D^{\alpha_{1}} f_{2} \cdots D^{\alpha_{l}} f_{2}\right|<\varepsilon \text { on } U
$$

Case $r^{\prime \prime}=0$. Put

$$
\begin{aligned}
U & =\left\{x \in \mathbf{R}^{n}:|f(x)|<\varepsilon(x) / 2\right\} \\
U^{\prime} & =\left\{(x, t) \in U \times \mathbf{R}:\left(f^{2}(x)+t^{2}\right)^{1 / 2}<\varepsilon(x)\right\} .
\end{aligned}
$$

Then $U^{\prime}$ is an open semialgebraic set containing $f^{-1}(0) \times 0$. Hence, by Argument 2.1 , for arbitrarily small $e, U^{\prime}$ contains the graph of $e(x)^{1 / 2}$ on $U$, consequently

$$
\left(f^{2}(x)+e(x)\right)^{1 / 2}<\varepsilon(x) \text { on } U \text {. }
$$

Thus Case $r^{\prime \prime}=0$ is proved.

Case $r^{\prime \prime}>0$. By Case $r^{\prime \prime}=0$ and the equality

$$
f_{2}^{r^{\prime \prime}-l+1} D^{\alpha_{1}} f_{2} \cdots D^{\alpha_{l}} f_{2}=f_{2} \prod_{i=1}^{l} f_{2}^{\left|\alpha_{i}\right|-1} D^{\alpha_{i}} f_{2}
$$

it is enough to prove globally

$$
\left|f_{2}^{\left|\alpha_{i}\right|-1} D^{\alpha_{i}} f_{2}\right| \leqslant C_{2}+|x|^{2 k_{2}}
$$

for each $i$, some $C_{2}, k_{2}$ and arbitrarily small $e$ with $k^{2 k} \leqslant C$. Now

$$
\left|D^{\alpha_{i}} f_{2}\right| \leqslant C \sum_{\beta_{1}+\cdots+\beta_{k}=\alpha_{i}}^{\substack{\beta_{j}>0}}\left|\left(f^{2}+e\right)^{1 / 2-k} D^{\beta_{1}}\left(f^{2}+e\right) \cdots D^{\beta_{k}}\left(f^{2}+e\right)\right|
$$

for some constant $C>0$. Hence we only need

$$
\left|\left(f^{2}+e\right)^{\left|\beta_{j}\right| / 2-1} D^{\beta_{j}}\left(f^{2}+e\right)\right|<C_{2}+|x|^{2 k_{2}} .
$$

But this is trivial if $\left|\beta_{j}\right|>1$, and if $\left|\beta_{j}\right|=1$ this follows from the inequality $\left|D^{\beta_{j}} e\right| \leqslant d_{0} e$ in the proof of Lemma 2.1. Hence the proof is completed.

Given $C_{1}, k_{1}, C_{2}$ and $k_{2}$ put $e_{1}=e_{C_{1}, k_{1}}$ and $e_{2}=e_{C_{2}, k_{2}}$. Let $\infty>r^{\prime} \leqslant r$ and let $\varphi$ be a polynomial on $\mathbf{R}$ such that $\varphi(0)=0, \varphi(1)=1$ and $\varphi^{\prime}=\cdots=\varphi^{\left(r^{\prime}\right)}=0$ at 0 and 1 if $r^{\prime} \geqslant 1$. Put

$$
\begin{aligned}
& F_{1}=(|3-f-| f-1||+3-f-|f-1|) / 4 \\
& F_{2}=\left(\left\{\left(3-f-\left\{(f-1)^{2}+e_{1}\right\}^{1 / 2}\right)^{2}+e_{2}\right\}^{1 / 2}+3-f-\left\{(f-1)^{2}+e_{1}\right\}^{1 / 2}\right) / 4 .
\end{aligned}
$$

Then $\varphi\left(F_{1}\right)$ is a $C^{r^{\prime}}$ Nash function such that $\varphi=0$ on $\{f \geqslant 2\}$ and $=1$ on $\{f \leqslant 1\}$. 
LEMMA 2.3. Choosing small $e_{1}$ and $e_{2}$ we can approximate $\varphi\left(F_{1}\right)$ by $\varphi\left(F_{2}\right)$ in the $C^{r^{\prime}}$ topology.

Proof. At present fix $e_{1}$. Put

$$
F_{3}=\left\{\left|3-f-\left((f-1)^{2}+e_{1}\right)^{1 / 2}\right|+3-f-\left((f-1)^{2}+e_{1}\right)^{1 / 2}\right\} / 4 .
$$

Then $\varphi\left(F_{3}\right)$ is a $C^{r^{\prime}}$ Nash function and Lemma 2.2 tells us $\varphi\left(F_{2}\right) \rightarrow \varphi\left(F_{2}\right)$ in the $C^{r^{\prime}}$ topology as $C_{2}$ and $k_{2} \rightarrow \infty$ satisfying $k_{2}^{2 k_{2}} \leqslant C_{2}$. Hence it suffices to prove $\varphi\left(F_{3}\right) \rightarrow \varphi\left(F_{1}\right)$ in the $C^{r^{\prime}}$ topology as $C_{1}$ and $k_{1} \rightarrow \infty$ satisfying $k_{1}^{2 k_{1}} \leqslant C_{1}$.

The case $r^{\prime}=0$ follows from Lemma 2.2. So assume $r^{\prime}>0$. We want an inequality

$$
\left|D^{\alpha} \varphi\left(F_{3}\right)-D^{\alpha} \varphi\left(F_{1}\right)\right| \leqslant \varepsilon
$$

for small $e_{1}$, where $\alpha$ is a multi-index with $0<|\alpha| \leqslant r^{\prime}$ and $\varepsilon$ is a given function of the same form as $e$. If $|\alpha|>0$ we have, like in the proof of Lemma 2.3,

$$
\begin{aligned}
& D^{\alpha} \varphi\left(F_{3}\right)=\left\{\begin{array}{l}
0 \text { on } Y_{3}, \\
\varphi^{\prime}\left(F_{3}\right) D^{\alpha} F_{3}+\varphi^{\prime \prime}\left(F_{3}\right) \sum_{\substack{\beta+\gamma=\alpha \\
\beta, \gamma \neq 0}} D^{\beta} F_{3} D^{\gamma} F_{3}+\cdots
\end{array} \text { outside } Y_{3},\right. \\
& D^{\alpha} \varphi\left(F_{1}\right)=\left\{\begin{array}{l}
0 \quad \text { on } Y_{1} \cup Y_{2}, \\
\varphi^{\prime}\left(F_{1}\right) D^{\alpha} F_{1}+\varphi^{\prime \prime}\left(F_{1}\right) \sum_{\substack{\beta+\gamma=\alpha \\
\beta, \gamma \neq 0}} D^{\beta} F_{1} D^{\gamma} F_{1}+\cdots
\end{array} \text { outside } Y_{1} \cup Y_{2},\right.
\end{aligned}
$$

where $Y_{1}=\{f=1\}, Y_{2}=\{f=2\}$, and $Y_{3}=\left\{3=f+\left((f-1)^{2}+e_{1}\right)^{1 / 2}\right\}=\{f$ $\left.=2-e_{1} / 4\right\}$. Now Argument 2.1 says that for sufficiently small $e_{1}, Y_{3}$ is contained in a given semialgebraic neighborhood of $Y_{2}$. Hence for (1) we only need to find open semialgebraic neighborhoods $U_{1}$ and $U_{2}$ of $Y_{1}$ and $Y_{2}$, respectively, and $C_{10}, k_{10}$ such that for each $\alpha_{1}, \ldots, \alpha_{l}>0$ with $\left|\alpha_{1}\right|+\cdots+\left|\alpha_{l}\right| \leqslant r^{\prime}$ and any $C_{1}>C_{10}$ and $k_{1}>k_{10}$ with $k_{1}^{2 k_{1}} \leqslant C_{1}$,

$$
\begin{gathered}
\left|\varphi^{(l)}\left(F_{3}\right) D^{\alpha_{1}} F_{3} \cdots D^{\alpha_{l}} F_{3}\right|<\varepsilon \text { on } U_{1} \cup U_{2}-Y_{3}, \\
\left|\varphi^{(l)}\left(F_{1}\right) D^{\alpha_{1}} F_{1} \cdots D^{\alpha_{l}} F_{1}\right|<\varepsilon \text { on } U_{1} \cup U_{2}-Y_{1} \cup Y_{2}, \\
\left|\varphi^{(l)}\left(F_{3}\right) D^{\alpha_{1}} F_{3} \cdots D^{\alpha_{l}} F_{3}-\varphi^{(l)}\left(F_{1}\right) D^{\alpha_{1}} F_{1} \cdots D^{\alpha_{l}} F_{1}\right|<\varepsilon \\
\text { on } \mathbf{R}^{n}-U_{1} \cup U_{2} .
\end{gathered}
$$

Here we can replace $F_{1}$ and $F_{3}$ by

$$
F_{10}=(3-f-|f-1|) / 2 \text { and } F_{30}=\left(3-f-\left((f-1)^{2}+e_{1}\right)^{1 / 2}\right) / 2 \text {, }
$$

respectively, because

$$
\begin{aligned}
& F_{1}(x)= \begin{cases}F_{10}(x) & \text { if } F_{10}(x)>0 \\
0 & \text { otherwise }\end{cases} \\
& F_{3}(x)= \begin{cases}F_{30}(x) & \text { if } F_{30}(x)>0 \\
0 & \text { otherwise }\end{cases}
\end{aligned}
$$


and because $F_{10}(x)>0$ if and only if $F_{30}(x)>0$ for $x \in \mathbf{R}^{n}-U_{1} \cup U_{2}$ and small $e_{1}$. Let $(2)_{0},(3)_{0}$ and $(4)_{0}$ denote the respective replaced inequalities.

At first (3) $)_{0}$ is trivial for some small $U_{1}$ and $U_{2}$ because

$$
\varphi^{(l)}(t)=t^{r^{\prime}-l+1}(t-1)^{r^{\prime}-l+1} \psi(t)
$$

for some polynomial $\psi$. Next Lemma 2.1 implies $F_{30} \rightarrow F_{10}$ on $\mathbf{R}^{n}-U_{1}$ in the $C^{r^{\prime}}$ topology as $C_{1}$ and $k_{1} \rightarrow \infty$ satisfying $k_{1}^{2 k_{1}} \leqslant C_{1}$, which, together with (3) 0 , proves $(2)_{0}$ on $U_{2}$ and $(4)_{0}$. (2) $)_{0}$ on $U_{1}$ is reduced, in the same way as the proof of Lemma 2.2 , to

$$
\left|f^{r^{\prime}-l+1} D^{\alpha_{1}} f_{1} \cdots D^{\alpha_{l}} f_{1}\right|<\varepsilon \text { on } U_{1}
$$

where $f_{1}=\left((f-1)^{2}+e_{1}\right)^{1 / 2}$. But this is the same as an inequality desired in the proof of Lemma 2.2. Hence the lemma is proved.

Let $X \subset \mathbf{R}^{n}$ be an algebraic set, $I$ the ideal of $\mathbf{R}\left[x_{1}, \ldots, x_{n}\right]$ defined by $X$, namely, consisting of polynomials vanishing on $X$, and $h$ the square sum of finite generators of $I$. Put $f=h^{r^{\prime}} / e_{3}$, where $C_{3}, k_{3}>1$ and $e_{3}=e_{C_{3}, k_{3}}$. Let $e_{1}, e_{2}, r^{\prime}, \varphi, F_{1}$ and $F_{2}$ be the same as stated just before Lemma 2.3.

Proposition 2.4. $\varphi\left(F_{1}\right)$ and $\varphi\left(F_{2}\right)$ are $C^{r^{\prime}}$ and $C^{\omega}$ Nash functions respectively. Let $U$ be a semialgebraic neighborhood of $X$. Then, for small $e_{3}, \varphi\left(F_{1}\right)=0$ outside $U$ and $=1$ in another neighborhood. Fix $e_{3}$. Then $\varphi\left(F_{2}\right)$ is an approximation of $\varphi\left(F_{1}\right)$ in the $C^{r^{\prime}}$ topology for small $e_{1}$ and $e_{1}$.

Proof. The first statement is clear by definition; the second follows if we can choose $e_{3}$ so that $U \supset\left\{h<2 e_{3}\right\}$, but this is possible by Argument 2.1; and the last is Lemma 2.3 .

Proposition 2.5. Let $Y \subset X$ be a connected component of $X-\operatorname{Sing} X$ ( $=$ the set of singular points of $X$ ) and let $V$ be a semialgebraic neighborhood of $X-Y$ in $\mathbf{R}^{n}$. Let $g$ be a $C^{r}$ Nash function on $\mathbf{R}^{n} r^{\prime}$-flat on $Y$. Then $g \varphi\left(F_{1}\right) \rightarrow 0$ on $\mathbf{R}^{n}-V$ in the $C^{r^{\prime}}$ topology as $C_{3}, k_{3} \rightarrow \infty$ satisfying $k_{3}^{2 k_{3}} \leqslant C_{3}$.

Proof. Let $\varepsilon \in N^{\omega}\left(\mathbf{R}^{n}\right)$ be of the same form as $e$ and $\alpha$ a multi-index with $|\alpha| \leqslant r^{\prime}$. Then we have to see

$$
\left|D^{\alpha}\left(g \varphi\left(F_{1}\right)\right)\right| \leqslant \varepsilon \quad \text { on } \mathbf{R}^{n}-V
$$

for large $C_{3}$ and $k_{3}$ with $k_{3}^{2 k_{3}} \leqslant C_{3}$. We will reduce (1) to plainer inequalities. As $\varphi\left(F_{1}\right)=0$ outside $W=\{f \leqslant 2\}$, it suffices to consider (1) on $W-V$. Moreover we can replace (1) by

$$
\left|D^{\alpha}(g \varphi(2-f))\right| \leqslant \varepsilon \quad \text { on } W-V
$$

because of

$$
\left|D^{\alpha}(g \varphi(2-f))\right| \geqslant\left|D^{\alpha}\left(g \varphi\left(F_{1}\right)\right)\right| \text { globally. }
$$


Now we have

$$
\begin{aligned}
D^{\alpha}(g \varphi(2-f))= & \sum_{\beta+\gamma=\alpha} D^{\beta} g D^{\gamma} \varphi(2-f), \\
D^{\gamma} \varphi(2-f)= & \varphi^{\prime}(2-f) D^{\gamma}(2-f)+\varphi^{\prime \prime}(2-f) \\
& \times \sum_{\substack{\delta+\zeta=\gamma \\
\delta, \zeta \neq 0}} D^{\delta}(2-f) D^{\zeta}(2-f)+\cdots
\end{aligned}
$$

if $|\gamma|>0$. Hence (2) follows from

$$
\left|D^{\beta} g D^{\alpha_{1}} f \cdots D^{\alpha_{l}} f\right| \leqslant \varepsilon \text { on } W-V,
$$

where $|\beta|+\left|\alpha_{1}\right|+\cdots+\left|\alpha_{l}\right| \leqslant r^{\prime}$, because $\varphi^{\prime}(2-f), \ldots, \varphi^{\left(r^{\prime}\right)}(2-f)$ are bounded on $W$. Furthermore (3) can be reduced to

$$
\left|D^{\beta} g D^{\alpha_{1}} H \cdots D^{\alpha_{l}} H D^{\gamma_{1}} e_{3} \cdots D^{\gamma_{k}} e_{3} / e_{3}^{k+1}\right| \leqslant \varepsilon \quad \text { on } W-V
$$

by an easy calculation, where $|\beta|+\left|\alpha_{1}\right|+\cdots+\left|\alpha_{l}\right|+\left|\gamma_{1}\right|+\cdots+\left|\gamma_{k}\right| \leqslant r^{\prime}$ and $H=h^{r^{\prime}}$. Here if $l=0$, then $k=-1$. Recall the inequality $\left|D^{\gamma_{i}} e_{3}\right| \leqslant d e$ for some constant $d$ in the proof of Lemma 2.1, by which we only need to prove

$$
\left|D^{\beta} g D^{\alpha_{1}} H \cdots D^{\alpha_{l}} H / H\right| \leqslant \varepsilon \text { on } W-V-Y,
$$

where $|\beta|+\left|\alpha_{1}\right|+\cdots+\left|\alpha_{l}\right| \leqslant r^{\prime}$ and $l \geqslant 1$, and

$$
\left|D^{\beta} g\right| \leqslant \varepsilon \text { on } W-V
$$

where $|\beta| \leqslant r^{\prime}$. Here we used the inequality $H \leqslant 2 e_{3}$ on $W$ and the hypothesis $D^{\beta} g=0$ on $Y$.

Consider the sets

$$
\begin{aligned}
Z & =\left\{x \in \mathbf{R}^{n}:\left|D^{\beta} g D^{\gamma_{1}} H \cdots D^{\gamma_{l}} H(x)\right| \leqslant \varepsilon(x) H(x)\right\}, \\
Z^{\prime} & =\left\{x \in \mathbf{R}^{n}:\left|D^{\beta} g(x)\right| \leqslant \varepsilon(x)\right\} .
\end{aligned}
$$

Then they are semialgebraic and contain $Y$. Hence Argument 2.1 tells us that it suffices to prove $Z$ and $Z^{\prime}$ are neighborhoods of $Y$. That is trivial for $Z^{\prime}$. For $Z$, let $x_{0}$ be a point of $Y$ and consider a small neighborhood of $x_{0}$. We can obtain a $C^{\infty}$ local coordinate system $(y, z)=\left(y_{1}, \ldots, y_{m}, z_{m+1}, \ldots, z_{n}\right)$ around $x_{0}$ such that $(y, z)$ $=0$ at $x_{0}$ and $h(y, z)=y_{1}^{2}+\cdots+y_{m}^{2}$ and $Y=\left\{y_{1}=\cdots=y_{m}=0\right\}$ (see the proof of Lemma 4.11 in [10]). By hypothesis we have

$$
\left|D_{x}^{\beta} g(y, z)\right| \leqslant d^{\prime}|y|^{r^{\prime}+1-|\beta|}
$$

in a neighborhood of 0 for some constant $d^{\prime}$. Hence it follows

$$
\left|D_{x}^{\beta} g D_{x}^{\alpha_{1}} H \cdots D_{x}^{\alpha_{l}} H(y, z)\right| \leqslant d^{\prime \prime}|y|^{r^{\prime}+1-|\beta|+2 r^{\prime}-\left|\alpha_{1}\right|+\cdots+2 r^{\prime}-\left|\alpha_{l}\right|} \leqslant d^{\prime \prime}|y|^{2 r^{\prime}+1}
$$

in a neighborhood of 0 for some constant $d^{\prime \prime}$. Thus $Z$ contains a neighborhod of $x_{0}$, which completes the proof.

3. The approximation theorem for Nash mappings. Assume $r<\infty$ in this section. In [2] Efroymson stated Corollary 2 in the $C^{0}$ topology, and he gave a key lemma to it. For the proof of Theorem 1 we shall need the lemma in a more general form (Lemma 3.1). But, unfortunately, [2] has several mistakes in the proofs. So we shall give a complete proof. 
Let $h \in N^{\omega}\left(\mathbf{R}^{n}\right), X=h^{-1}(0), U \subset X$ a connected $C^{\omega}$ Nash manifold open in $X$ and $g \in N^{\omega}(U)$. A minimal polynomial $P(z, x)$ for $g$ means a polynomial in $n+1$ variables such that $\left.P(z, x)\right|_{\mathbf{R} \times U} \not \equiv 0, P(g(x), x) \equiv 0$ on $U$ and the degree in $z$ is minimal. We say the pair $(g, P)$ has Property (A1) if $P^{-1}(0) \cap\left(\partial P^{-1} / \partial z\right)(0) \cap \mathbf{R} \times$ $U=\varnothing, P$ is of constant degree in $z$ at every point of $U$ and $\left\{P^{-1}(0) \cup\right.$ $\left.\left(\partial P^{-1} / \partial z\right)(0)\right\} \cap \mathbf{R} \times U$ is the disjoint union of the graphs of $C^{\omega}$ Nash functions on $U$. Moreover, by induction, if the pair of each $C^{\omega}$ Nash function on $U$ whose graph is contained in $\left(\partial P^{-1} / \partial z\right)(0)$ and some minimal polynomial for it has Property (A $k-1)$, then we say $(g, P)$ has Property $(\mathrm{A} k)$ for $k>1$. Write simply Property (A $m$ ) as Property (A) for $m=$ degree in $z$ of $P$. Let $(g, P)$ have Property (A). Then we say $(g, P)$ is of height 0 if $\left(\partial P^{-1} / \partial z\right)(0) \cap \mathbf{R} \times U=\varnothing$ and, inductively, $(g, P)$ is of height $l$ if it is not of height $l-1$ and the pair of each $C^{\omega}$ Nash function on $U$ defined by $\left(\partial P^{-1} / \partial z\right)(0)$ and some minimal polynomial for it is of height $\leqslant l-1$. It is clear that if $(g, P)$ has Property (A1) we can extend $g$ uniquely to some semialgebraic neighborhood of $U$ in $\mathbf{R}^{n}$ satisfying $P(g(x), x) \equiv 0$. We write the extension as $\tilde{g}_{P}$. We will not specify the domain of $\tilde{g}_{P}$.

LEMMA 3.1. Let $D \subset \mathbf{R}^{n}$ be a closed semialgebraic set contained in $U$. Assume the pair of $g \in N^{\omega}(U)$ and a polynomial $P$ has Property (A). Then there exists a closed semialgebraic neighborhood $\tilde{D}$ of $D$ in $\mathbf{R}^{n}$ such that $\tilde{g}_{P}$ is defined on $\tilde{D}$ and $\left.\tilde{g}_{P}\right|_{\tilde{D}}$ can be approximated in the $C^{\omega}$ topology by the restriction to $\tilde{D}$ of a $C^{\omega}$ Nash function on $\mathbf{R}^{n}$.

Proof. At first we can assume $D$ is connected for the following reason. By Theorem 1 in [9] $U$ is $C^{\omega}$ Nash diffeomorphic to the interior of a compact $C^{\omega}$ Nash manifold possibly with boundary. Hence consider in place of $D$ the compact manifold-an open collar. Next applying the Mostowski separation theorem [6] to $D$ and $X-U$, we have $h_{1} \in N^{\omega}\left(\mathbf{R}^{n}\right)$ such that $h_{1}>0$ on $D$ and $h_{1}<0$ on $X-U$. Put $D_{1}=\left\{x \in X: h_{1}(x) \geqslant 0\right\}$ and let $D_{2}$ be the connected component of $D_{1}$ containing $D$. Once more by the separation theorem there exists $h_{2} \in N^{\omega}\left(\mathbf{R}^{n}\right)$ such that $h_{2}>0$ on $D_{2}$ and $h_{2}<0$ on $D_{1}-D_{2}$. Hence

$$
D_{2}=\left\{x \in X: h_{1}(x) \geqslant 0 \text { and } h_{2}(x) \geqslant 0\right\} .
$$

For $C$ and $k>1$, put

$$
D_{C, k}=\left\{x \in \mathbf{R}^{n}: h_{0}(x)=e_{C, k}(x)-h^{2}(x) \geqslant 0, h_{1}(x) \geqslant 0, h_{2}(x) \geqslant 0\right\} .
$$

We shall define $\tilde{D}$ so that it will be contained in the interior of this $D_{C, k}$ for some large $C$ and $k$. Here we remark every semialgebraic neighborhood of $D_{2}$ contains $D_{C, k}$ for some $C$ and $k$ by Argument 2.1 .

The present subject is to prove the lemma in the $C^{0}$ topology by induction on the height of $(g, P)$. Let $P(z, x)=a_{m} z^{m}+\cdots+a_{0}, a_{m} \neq 0$. Then the minimality and the constancy of degree of $P$ show $a_{m}>0$ on $U$ or $a_{m}<0$ on $U$, hence assume $a_{m}>0$ on $U$.

Case height $=0$. By definition and by assumption, $\partial P / \partial z$ and $a_{m}$ are positive on $\mathbf{R} \times U$ and $D_{C, k}$ for some $C, k$ respectively. Hence we can choose $D_{C, k}$ so that $\partial P / \partial z>0$ on $\mathbf{R} \times D_{C, k}$ and, consequently, $\tilde{g}_{P}$ is defined on $D_{C, k}$. Fix such $C$ and $k$. By Argument 2.1 we have polynomials $\varphi_{1}(x)$ and $\varphi_{2}(x)$ on $\mathbf{R}^{n}$ such that $\varphi_{2}>\tilde{g}_{P}>\varphi_{1}$ 
on $D_{C, k}$ and $\varphi_{2}>\varphi_{1}$ on $\mathbf{R}^{n}$. Clearly $P\left(\varphi_{1}(x), x\right)<0$ and $P\left(\varphi_{1}(x), x\right)>0$ on $D_{C, k}$. Put

$$
\begin{gathered}
H_{C^{\prime}, k^{\prime}}=\sum_{i=0}^{2}\left(\left(h_{i}^{2}+e_{C^{\prime}, k^{\prime}}\right)^{1 / 2}-h_{i}\right), \\
P_{C^{\prime}, k^{\prime}}(z, x)=P(z, x)+\left(u_{2}+u_{1}\right)\left(z-\varphi_{1}\right) H_{C^{\prime}, k^{\prime}} /\left(\varphi_{2}-\varphi_{1}\right)-u_{1} H_{C^{\prime}, k^{\prime}}
\end{gathered}
$$

for polynomials $u_{1}$ and $u_{2}$ on $\mathbf{R}^{n}$. Then for some large $u_{1}, u_{2}$ and for any $C^{\prime}, k^{\prime}$ we have

$$
\begin{gathered}
P_{C^{\prime}, k^{\prime}}\left(\varphi_{1}(x), x\right)=P\left(\varphi_{1}(x), x\right)-u_{1} H_{C^{\prime}, k^{\prime}}<0 \quad \text { on } \mathbf{R}^{n}, \\
P_{C^{\prime}, k^{\prime}}\left(\varphi_{2}(x), x\right)=P\left(\varphi_{2}(x), x\right)+u_{2} H_{C^{\prime}, k^{\prime}}>0 \quad \text { on } \mathbf{R}^{n}, \\
\frac{\partial P_{C^{\prime}, k^{\prime}}}{\partial z}(z, x)=\frac{\partial P}{\partial z}(z, x)+\frac{\left(u_{2}+u_{1}\right) H_{C^{\prime}, k^{\prime}}}{\varphi_{2}-\varphi_{1}}>0 \quad \text { for } \varphi_{1}(x) \leqslant z \leqslant \varphi_{2}(x) .
\end{gathered}
$$

Fix such $u_{1}$ and $u_{2}$. Then $P_{C^{\prime}, k^{\prime}}(z, x)=0$ has the unique solution $g_{C^{\prime}, k^{\prime}}(x)$ on $\mathbf{R}^{n}$ such that $\varphi_{2}>g_{C^{\prime}, k^{\prime}}>\varphi_{1}$. Trivially $g_{C^{\prime}, k^{\prime}}$ is of class $C^{\omega} \mathrm{Nash}$; and Proposition 3 in [2] whose proof is easy and complete implies $\left.\left.g_{C^{\prime}, k^{\prime}}\right|_{D_{C, k}} \rightarrow \tilde{g}_{P}\right|_{D_{C, k}}$ in the $C^{0}$ topology as $C^{\prime}, k^{\prime} \rightarrow \infty$, because the coefficients of the $z$-polynomial $P-P_{C^{\prime}, k^{\prime}}$ coriverge to 0 on $D_{C, k}$ in the $C^{0}$ topology as $C^{\prime}, k^{\prime} \rightarrow \infty$ by Lemma 2.2. Thus we have proved the case height $=0$.

By induction assume height $(g, P)=l>0$ and the lemma in the $C^{0}$ topology in the case height $\leqslant l-1$. We define $\varphi_{1} \in N^{\omega}\left(\mathbf{R}^{n}\right)$ like in the case height $=0$ as follows. If every root of $(\partial P / \partial z)(z, x)$ on $U$ is larger than $g(x)$, then let $\varphi_{1}$ be a polynomial on $\mathbf{R}^{n}$ such that $g>\varphi_{1}$ on $D_{2}$. In the other case let $g^{\prime} \in N^{\omega}(U)$ be the largest root of $(\partial P / \partial z)(z, x)$ with $g>g^{\prime}$ on $U$. Then, by definitions of Property (A) and height we have a minimal polynomial $P^{\prime}$ for $g^{\prime}$ such that $\left(g^{\prime}, P^{\prime}\right)$ has Property (A) and is of height $<l$. Hence, by the induction hypothesis there exist $\varphi_{1}^{\prime} \in N^{\omega}\left(\mathbf{R}^{n}\right)$ such that $\left.\varphi_{1}^{\prime}\right|_{D_{2}}$ is an approximation of $\left.g^{\prime}\right|_{D_{2}}$ in the $C^{0}$ topology. Choose a positive $C^{\omega}$ Nash function $\varphi_{1}^{\prime \prime}$ on $\mathbf{R}^{n}$ so small that $2 \varphi_{1}^{\prime \prime}<g-g^{\prime}$ on $D_{2}$, and take the above approximation closely enough. Then $\varphi_{1}=\varphi_{1}^{\prime}+\varphi_{1}^{\prime \prime}$ satisfies

(1) $\varphi_{1}<g$ on $D_{2}, \quad \frac{\partial P}{\partial z}(z, x)>0$ for $\varphi_{1}(x) \leqslant z \leqslant g(x)$ and $x \in D_{2}$.

We remark (1) holds true in the previous case too. We also define $\varphi_{2} \in N^{\omega}\left(\mathbf{R}^{n}\right)$ so that

(2) $\varphi_{2}>g$ on $D_{2}, \quad \frac{\partial P}{\partial z}(z, x)>0$ for $g(x) \leqslant z \leqslant \varphi_{2}(x)$ and $x \in D_{2}$.

If necessary, adding to $\varphi_{2}$ a $C^{\omega}$ Nash function of the form $u H_{C^{\prime}, k^{\prime}}$ for large $u \in N^{\omega}\left(\mathbf{R}^{n}\right), C^{\prime}$ and $k^{\prime}$, we can assume, moreover, $\varphi_{1}<\varphi_{2}$ on $\mathbf{R}^{n}$. Hence gathering (1) and (2) we have

$$
\begin{gathered}
\varphi_{1}<\varphi_{2} \text { on } \mathbf{R}^{n}, \quad \varphi_{1}<g<\varphi_{2} \text { on } D_{2}, \\
\frac{\partial P}{\partial z}(z, x)>0 \text { for } \varphi_{1}(x) \leqslant z \leqslant \varphi_{2}(x) \text { and } x \in D_{2} .
\end{gathered}
$$


As the second and last inequalities in (3) hold in a semialgebraic neighborhood of $D_{2}$ in $\mathbf{R}^{n}$, (3) implies for arbitrarily large $C$ and $k$

$$
\begin{gathered}
\varphi_{1}<\varphi_{2} \text { on } \mathbf{R}^{n}, \quad \varphi_{1}<\tilde{g}_{P}<\varphi_{2} \text { on } D_{C, k} \\
\frac{\partial P}{\partial z}(z, x)>0 \quad \text { for } \varphi_{1}(x) \leqslant z \leqslant \varphi_{2}(x) \text { and } x \in D_{C, k} .
\end{gathered}
$$

Hence the case height $=l$ follows in exactly the same way as the case height $=0$. Thus, in any case, $\left.\tilde{g}_{P}\right|_{D_{C, k}}$ is approximated by $\left.\tilde{g}_{C^{\prime}, k^{\prime}}\right|_{D_{C, k}}$ in the $C^{0}$ topology where $g_{C^{\prime}, k^{\prime}}$ is a root of $P_{C^{\prime}, k^{\prime}}$ which is of the form

$$
P_{C^{\prime}, k^{\prime}}=P+a_{1}^{\prime} H_{C^{\prime}, k^{\prime}} z+a_{0}^{\prime} H_{C^{\prime}, k^{\prime}},
$$

where $a_{0}^{\prime}, a_{1}^{\prime} \in N^{\omega}\left(\mathbf{R}^{n}\right)$.

Let $0<r$ and $\tilde{D}$ be a closed semialgebraic neighborhood of $D$ in $\mathbf{R}^{n}$ contained in the interior of $D_{C, k}$. What is left is to show $\left.\left.g_{C^{\prime}, k^{\prime}}\right|_{\tilde{D}} \rightarrow \tilde{g}_{P}\right|_{\tilde{D}}$ in the $C^{r}$ topology as $C^{\prime}$ and $k^{\prime} \rightarrow \infty$ satisfying $k^{\prime 2 k^{\prime}} \leqslant C^{\prime}$. We will work by induction on $r$. So assume this convergence in the $C^{r-1}$ topology. By Lemma 2.1 we already know $H_{C^{\prime}, k^{\prime}} \rightarrow 0$ on $\tilde{D}$ in the $C^{r}$ topology as $C^{\prime}$ and $k^{\prime} \rightarrow \infty$ satisfying $k^{\prime 2 k^{\prime}} \leqslant C^{\prime}$.

Let $\alpha$ be a multi-index with $|\alpha|=r$. Trivially we have

$$
\begin{aligned}
0= & D^{\alpha}\left\{P\left(\tilde{g}_{P}(x), x\right)\right\} \\
= & \left(D^{\alpha} P\right)\left(\tilde{g}_{P}, x\right)+\frac{\partial P}{\partial z}\left(\tilde{g}_{P}, x\right) D^{\alpha} \tilde{g}_{P} \\
& +\sum_{\substack{\beta+\gamma=\alpha \\
\beta, \gamma>0}} \frac{\partial^{2} P}{\partial z^{2}}\left(\tilde{g}_{P}, x\right) D^{\beta} \tilde{g}_{P} D^{\gamma} \tilde{g}_{P}+\cdots, \\
0= & D^{\alpha}\left\{P_{C^{\prime}, k^{\prime}}\left(g_{C^{\prime}, k^{\prime}}(x), x\right)\right\} \\
= & \left(D^{\alpha} P_{C^{\prime}, k^{\prime}}\right)\left(g_{C^{\prime}, k^{\prime}}, x\right)+\frac{\partial P_{C^{\prime}, k^{\prime}}}{\partial z}\left(g_{C^{\prime}, k^{\prime}}, x\right) D^{\alpha} g_{C^{\prime}, k^{\prime}} \\
& +\underset{\substack{\beta+\gamma=\alpha \\
\beta, \gamma>0}}{ } \frac{\partial^{2} P_{C^{\prime}, k^{\prime}}}{\partial z^{2}}\left(g_{C^{\prime}, k^{\prime}}, x\right) D^{\beta} g_{C^{\prime}, k^{\prime}} D^{\gamma} g_{C^{\prime}, k^{\prime}}+\cdots .
\end{aligned}
$$

By $(3)^{\prime}$,

$$
\frac{\partial P}{\partial z}\left(\tilde{g}_{P}(x), x\right)>0 \text { for } x \in \tilde{D}
$$

Hence, by Argument $2.1,1 /\left(\partial P_{C^{\prime}, k^{\prime}} / \partial z\right)\left(g_{C^{\prime}, k^{\prime}}(x), x\right)$ is a $C^{\omega}$ Nash function on an open semialgebraic neighborhood of $\tilde{D}$ for arbitrarily large $C^{\prime}$ and $k^{\prime}$, and $1 /\left(\partial P_{C^{\prime}, k^{\prime}} / \partial z\right)\left(g_{C^{\prime}, k^{\prime}}(x), x\right) \rightarrow 1(\partial P / \partial z)\left(\tilde{g}_{P}(x), x\right)$ on $\tilde{D}$ in the $C^{0}$ topology as $C^{\prime}$ and $k^{\prime} \rightarrow \infty$. Therefore the above equalities and the induction hypothesis imply $D^{\alpha} g_{C^{\prime}, k^{\prime}} \rightarrow D^{\alpha} \tilde{g}_{P}$ on $\tilde{D}$ in the $C^{0}$ topology as $C^{\prime}$ and $k^{\prime} \rightarrow \infty$ satisfying $k^{\prime 2 k^{\prime}} \leqslant C^{\prime}$, which completes the proof. 
3.2. Reduction of Theorem 1 to the case $M_{1}=\mathbf{R}$ and $M_{2}=\mathbf{R}$. Let $M_{2}$ be contained in $\mathbf{R}^{n_{2}}$. By Lemma 7 in [9] there exists a $C^{\omega}$ Nash tubular neighborhood $V_{2}$ of $M_{2}$ in $\mathbf{R}^{n_{2}}$ (i.e. $V_{2}$ is a $C^{\omega}$ Nash manifold and the projection $p_{2}: V_{2} \rightarrow M_{2}$ is of class $C^{\omega}$ Nash). If $F: M_{1} \rightarrow \mathbf{R}^{n_{2}}$ is a $C^{\omega}$ Nash approximation of $f$ in the $C^{r}$ topology such that $F\left(M_{1}\right) \subset V_{2}$, then $p_{2} \circ F$ is a required approximation. Hence we can assume $M_{2}=\mathbf{R}^{n_{2}}$ and, hence, $M_{2}=\mathbf{R}$.

As [9] pointed out, we can assume $M_{1}$ is closed in $\mathbf{R}^{n}$. Let $p_{1}: V_{1} \rightarrow M_{1}$ be a $C^{\omega}$ Nash tubular neighborhood of $M_{1}$ in $\mathbf{R}^{n}$. By the separation theorem and the argument just before Lemma 2.3 we have $\varphi \in N^{r}\left(\mathbf{R}^{n}\right)$ such that $\varphi=1$ on a neighborhood of $M_{1}$ and $\varphi=0$ on a neighborhood of $\mathbf{R}^{n}-V_{1}$. Consider $\left(f \circ p_{1}\right) \varphi$ $\in N^{r}\left(V_{1}\right)$. This is extensible to $\mathbf{R}^{n}$ and the restriction to $M_{1}$ is equal to $f$. Hence it suffices to approximate the extension by a $C^{\omega}$ Nash function on $\mathbf{R}^{n}$.

3.3. There exist a stratification $\left\{U_{i j}\right\}_{0 \leqslant i \leqslant n ; 1 \leqslant j \leqslant m_{i}}$ of $\mathbf{R}^{n}$, polynomials $h_{i k}$ on $\mathbf{R}^{n}$ for all $i$ and $k$ with $1 \leqslant k \leqslant i, f_{i j \alpha} \in N^{\omega}\left(U_{i j}\right)$ for all $i, j$ and $\alpha \in \mathbf{N}^{i}$ with $|\alpha| \leqslant r$, and minimal polynomials $P_{i j \alpha}$ for $f_{i j \alpha}$ such that the following is satisfied. (Here $\mathbf{N}^{0}$ means $\{0\}$.

(3.3.1) Each $U_{i j}$ is a connected $C^{\omega}$ Nash manifold of dimension $n-i$.

(3.3.2) For each $i, B_{i}=\bigcup_{i^{\prime} \geqslant i ; 1 \leqslant j \leqslant m_{i^{\prime}}} U_{i^{\prime} j}$ is an algebraic set and each $U_{i j}$ consists of nonsingular points of $B_{i}$.

(3.3.3) For each $i, j$ and all $k, h_{i k}$ vanishes on $U_{i j}$ and $\operatorname{grad} h_{i k}$ span the normal vector bundle of $U_{i j}$ in $\mathbf{R}^{n}$.

(3.3.4) $\left(f_{i j \alpha}, P_{i j \alpha}\right)$ have Property (A).

(3.3.5) For each $i$ and $j, f-\sum_{|\alpha| \leqslant r} \tilde{f}_{i j \alpha P_{i j \alpha}} h_{i}^{\alpha}$ is r-flat on $U_{i j}$, where $h_{i}^{\alpha}$ means $\prod_{k=1}^{i} h_{i k}^{\alpha_{k}}$ for $\alpha=\left(\alpha_{1}, \ldots, \alpha_{i}\right)$.

Proof of 3.3. As (3.3.5) requires $f_{i j 0}=\left.f\right|_{U_{i j}}$ for any $i$ and $j$, we define it so. We construct $U_{i j}, h_{i k}, f_{i j \alpha}$ and $P_{i j \alpha}$ by double induction on $i$ and $|\alpha|$. Let $0 \leqslant s \leqslant n$. Assume we already have an algebraic set $B_{s} \subset \mathbf{R}^{n}$ of codimension $s$, a stratification $\left\{U_{i j}\right\}_{0 \leqslant i<s ; 1 \leqslant j \leqslant m_{i}}$ of $\mathbf{R}^{n}-B_{s}, h_{i k}, f_{i j}$ and $P_{i j \alpha}$ for $0 \leqslant i<s$ such that (3.3.1)-(3.3.5) are satisfied (here $B_{i}$ in (3.3.2) is modified to be $\bigcup_{i^{\prime} \geqslant i ; 1 \leqslant j \leqslant m_{i^{\prime}}} U_{i^{\prime} j} \cup B_{s}$ and we put $\left.B_{0}=\mathbf{R}^{n}\right)$. We shall define an algebraic set $B_{s+1}\left(\subset B_{s}\right)$ of codimension $<n-s$ as the Zariski closure of $B_{s+1}^{1} \cup B_{s+1}^{2} \cup \cdots$, where $B_{s+1}^{t}$ will be semialgebraic sets defined one after another; and we shall let $U_{s j}, j=1, \ldots, m_{s}$, be the connected components of $B_{s}-B_{s+1}$ so that we shall be able to define $h_{s k}, f_{s j \alpha}$ and $P_{s j \alpha}$.

It is elementary to find polynomials $h_{s k}, 1 \leqslant k \leqslant s$, so that they vanish on $B_{s}$ and the set of points of $B_{s}$ where $\operatorname{grad} h_{s k}, 1 \leqslant k \leqslant s$, are linearly dependent is of dimension $<n-s$. Denote the set by $B_{s+1}^{1}$. Then (3.3.3) will be satisfied for whatever $U_{s j}$.

Let $E$ be the graph of $f$ on $B_{s}$. As $E$ is semialgebraic, it admits a semialgebraic stratification [5]. Hence we have a semialgebraic set $E^{\prime}(\subset E)$ of dimension $<n-s$ such that $E-E^{\prime}$ is a $C^{\omega}$ Nash manifold of dimension $n-s$. Let $E^{\prime \prime}\left(\subset E-E^{\prime}\right)$ be the set of points where the projection $p: E-E^{\prime} \rightarrow \mathbf{R}^{n}$ is not $C^{\infty} \operatorname{regular}\left(E^{\prime \prime}=\varnothing\right.$ if $r \geqslant 1)$. Then it is easy to see by the Tarski-Seidenberg Theorem that $E^{\prime \prime}$ is a semialgebraic set of dimension $<n-s$, and $B_{s}-p\left(E^{\prime}\right)-p\left(E^{\prime \prime}\right)$ is a $C^{\omega}$ Nash manifold on which $f$ is of class $C^{\omega}$. Put $B_{s+1}^{2}=p\left(E^{\prime}\right) \cup p\left(E^{\prime \prime}\right)$. 
We will define $f_{s j \alpha}$ and $P_{s j \alpha}$ by induction on $|\alpha|$.

Case $\alpha=0$. Let $W$ be a connected component of $B_{s}-B_{s+1}^{1}-B_{s+1}^{2}$ and $P_{W}$ a minimal polynomial for $f_{W}=\left.f\right|_{W}$. Apply the above argument to $P_{W}^{-1}(0)$. Then we have a semialgebraic set $W^{\prime} \subset W$ of dimension $<n-s$ such that $P_{W}^{-1}(0) \cap \mathbf{R} \times$ (each connected component of $W-W^{\prime}$ ) is the disjoint union of the graphs of $C^{\omega}$ Nash functions. Here we remark the minimality of $P_{W}$ implies

$$
\operatorname{dim}\left(P_{W}^{-1}(0) \cap \frac{\partial P^{-1}}{\partial z}(0) \cap \mathbf{R} \times W\right)<n-s .
$$

Hence, in the same way as above, we can choose a semialgebraic set $W^{\prime \prime} \subset W$ of dimension $<n-s$ so that

$$
P_{W}^{-1}(0) \cap \frac{\partial P^{-1}}{\partial z}(0) \cap \mathbf{R} \times\left(W-W^{\prime}-W^{\prime \prime}\right)=\varnothing
$$

and $\left(\partial P^{-1} / \partial z\right)(0) \cap \mathbf{R} \times\left(\right.$ each connected component of $\left.W-W^{\prime}-W^{\prime \prime}\right)$ is the disjoint union of the graphs of $C^{\omega}$ Nash functions. Considering minimal polynomials for these $C^{\omega}$ Nash functions and repeating this argument, we obtain a finite number of semialgebraic sets $W^{\prime}, W^{\prime \prime}, \ldots$ such that, for each connected component $U$ of $W-W^{\prime}-W^{\prime \prime}-\cdots,\left(\left.f\right|_{U}, P_{W}\right)$ has Property (A). Hence we define $P_{s j 0}=P_{W}$ if $U_{s j} \subset W-W^{\prime}-\cdots$ and we put

$$
B_{s+1 W}^{3}=W^{\prime} \cup W^{\prime \prime} \cup \cdots \text { and } B_{s+1}^{3}=\bigcup_{W} B_{s+1 W}^{3} .
$$

Case $|\alpha|=l>0$. By induction assume we already have semialgebraic sets $B_{s+1}^{3}, \ldots, B_{s+1}^{l+2}$ of dimension $<n-s$, the connected components $O_{j}, j=1,2, \ldots$, of $B_{s}-B_{s+1}^{1}-\cdots-B_{s+1}^{l+2}, f_{j \beta} \in N^{\omega}\left(O_{j}\right)$ for all $j$ and $\beta \in \mathbf{N}^{s}$ with $|\beta|<l$ and minimal polynomials $P_{j \beta}$ for $f_{j \beta}$ such that $\left(f_{j \beta}, P_{j \beta}\right)$ have Property (A) and, for each $j$, $F_{j l-1}=f-\Sigma_{|\beta|<1} \tilde{f}_{j \beta P_{j \beta}} h_{s}^{\beta}$ is $(l-1)$-flat on $O_{j}$. Then there exist $f_{j \alpha} \in N^{\omega}\left(O_{j}\right)$ for each $j$ and all $\alpha$ with $|\alpha|=l$ such that $F_{j l-1}-\sum_{|\alpha|=l} \tilde{f}_{j \alpha} h_{s}^{\alpha}$ is $l$-flat on $O_{j}$ where $\tilde{f}_{j \alpha}$ are any $C^{\infty}$ extensions of $f_{i \alpha}$ for the following reason. Consider a local coordinate system of $\mathbf{R}^{m}$ of class $C^{\omega}$ Nash around each point of $O_{j}$ such that the system contains $h_{s 1}, \ldots, h_{s s}$. Then $O_{j}$ is a linear subspace in this coordinate system. Hence the existence of $\tilde{f}_{j \alpha}$ of class $C^{\infty}$ follows immediately, moreover the uniqueness of $f_{j \alpha}$ follows. This uniqueness, together with the fact that a derivative of a $C^{\omega}$ Nash function is of class $C^{\omega}$ Nash, implies that $f_{j \alpha}$ are $C^{\omega}$ Nash functions.

For all $j$ and $\alpha$ with $|\alpha|=l$, apply to $f_{j \alpha}$ the argument in the case $\alpha=0$. Then we obtain a semialgebraic set $B_{s+1}^{l+3}$ of dimension $<n-s$, the connected components $O_{j^{\prime}}^{\prime}, j^{\prime}=1,2, \ldots$, of $B_{s}-B_{s+1}^{1}-\cdots-B_{s+1}^{l+3}, f_{j^{\prime} \alpha} \in N^{\omega}\left(O_{j^{\prime}}^{\prime}\right)$ for all $j^{\prime}$ and $\alpha$ with $|\alpha|=l$ and minimal polynomials $P_{j^{\prime} \alpha}$ for $f_{j^{\prime} \alpha}$ such that $\left(f_{j^{\prime} \alpha}, P_{j^{\prime \alpha}}\right)$ have Property (A) and, for each $j^{\prime},\left.F_{j l-1}\right|_{O_{j^{\prime}}^{\prime}}-\sum_{|\alpha|=l} \tilde{f}_{j^{\prime} \alpha P_{j^{\prime} \alpha}} h_{s}^{\alpha}$ is $l$-flat on $O_{j^{\prime}}^{\prime}$ where $j$ is such that $O_{j} \supset O_{j^{\prime \prime}}^{\prime}$. If $|\alpha|<l$ we put $f_{j^{\prime} \alpha}=\left.f_{j \alpha}\right|_{O^{\prime}}, P_{j^{\prime} \alpha}=P_{j \alpha}$ for some $j$ with $O_{j} \supset O_{j^{\prime}}^{\prime}$. Hence we can define by induction $f_{s j \alpha}$ and $P_{s j \alpha}$ for all $\alpha$ with $|\alpha| \leqslant r$. Thus we have proved the statement for $s+1$ at the beginning of the proof. Therefore the proof is completed by induction on $s$. 
Let $0 \leqslant s \leqslant n$ and $\varepsilon$ be of the same form as $e$. Under the same notations as 3.3

3.4.s. For all $i$ and $j$ with $i \geqslant s$ there exist $H_{i j} \in N^{r}\left(\mathbf{R}^{n}\right)$ and arbitrarily small open semialgebraic neighborhoods $V_{i j} \subset V_{i j}^{\prime}$ of $U_{i j}$ such that

(3.4.s.1)

$$
\bar{V}_{i j} \subset V_{i j}^{\prime} \cup \bar{U}_{i j}, \quad H_{i j}= \begin{cases}1 & \text { on } V_{i j}-W_{i+1}^{\prime}, \\ 0 & \text { on } \mathbf{R}^{n}-\left(V_{i j}^{\prime}-W_{i+1}\right),\end{cases}
$$

where $W_{i+1}=\bigcup_{i<i^{\prime} ; 1 \leqslant j^{\prime} \leqslant m_{i}} V_{i^{\prime} j^{\prime}}, W_{i+1}^{\prime}=\bigcup_{i<i^{\prime} ; 1 \leqslant j^{\prime} \leqslant m_{i^{\prime}}} V_{i^{\prime} j^{\prime}}^{\prime}$;

(3.4.s.2) we can approximate $H_{i j}$ by $C^{\omega}$ Nash functions on $\mathbf{R}^{n}$ in the $C^{r}$ topology;

(3.4.s.3) $\sum_{i \geqslant s ; 1 \leqslant j \leqslant m_{i}} H_{i j}=1$ on $W_{s}$; and

(3.4.s.4) for each $i$ and $j, \Sigma_{|\alpha| \leqslant r} \tilde{f}_{i j \alpha P_{i j \alpha}} h_{i}^{\alpha} H_{i j}$ is an $\varepsilon$-approximation of $f H_{i j}$ of order $r$, i.e.

$$
\left|D^{\beta}\left\{f H_{i j}-\sum_{|\alpha| \leqslant r} \tilde{f}_{i j \alpha P_{i j \alpha}} h_{i}^{\alpha} H_{i j}\right\}\right| \leqslant \varepsilon \quad \text { on } \mathbf{R}^{n}
$$

for all $\beta$ with $|\beta| \leqslant r$.

Proof OF 3.4.s. We work by downward induction on $s$. If $s=n, 3.4$.s follows from Propositions 2.4 and 2.5 and (3.3.5). Hence assume 3.4. $s+1$. It suffices to consider 3.4.s on one $U_{s j}$ because we require, moreover, $V_{s j}^{\prime} \cap V_{s j^{\prime}}^{\prime}=\varnothing$ for $j \neq j^{\prime}$. Put

$$
G=1-\sum_{\substack{i>s \\ 1 \leqslant j^{\prime} \leqslant m_{i}}} H_{i j^{\prime}} \text { and } \rho=\sum_{|\alpha| \leqslant r} \tilde{f}_{s j \alpha P_{s j \alpha}} h_{s}^{\alpha} .
$$

Then $G=1$ on $\mathbf{R}^{n}-W_{s+1}^{\prime}$ by (3.4.s +1.1$), G=0$ on $W_{s+1}$ by $(3.4 . s+1.3)$ and $\rho-f$ is $r$-flat on $U_{s j}$ by (3.3.5). Apply the separation theorem to $U_{s j}-W_{s+1}$ and the complement of its small open semialgebraic neighborhood and apply Lemma 2.3 to the resultant separation function. Then there exists $H_{s j}^{\prime} \in N^{r}\left(\mathbf{R}^{n}\right)$ such that $H_{s j}^{\prime}=0$ outside a small semialgebraic neighborhood of $U_{s j}-W_{s+1}, H_{s j}^{\prime}=1$ on another one and we can approximate $H_{s j}^{\prime}$ by a $C^{\omega}$ Nash function on $\mathbf{R}^{n}$ in the $C^{r}$ topology. Hence, by Propositions 2.4 and 2.5 we have $H_{s j}^{\prime \prime} \in N^{r}\left(\mathbf{R}^{n}\right)$ such that $H_{s j}^{\prime \prime}=0$ outside an arbitrarily small open semialgebraic neighborhood of $B_{s}, H_{s j}^{\prime \prime}=1$ on another one, $H_{s j}^{\prime \prime}$ can be approximated by a $C^{\omega}$ Nash function on $\mathbf{R}^{n}$ in the $C^{r}$ topology, and $\rho G H_{s j}^{\prime} H_{s j}^{\prime \prime}$ is an $\varepsilon$-approximation of $f G H_{s j}^{\prime} H_{s j}^{\prime \prime}$ of order $r$. Therefore $H_{s j}=G H_{s j}^{\prime} H_{s j}^{\prime \prime}$, together with some arbitrarily small $V_{s j}$ and $V_{s j}^{\prime}$, satisfies 3.4.s. Clearly we can choose $V_{s j}^{\prime}$ 's so that $V_{s j}^{\prime} \cap V_{s j^{\prime}}^{\prime}=\varnothing$ for $j \neq j^{\prime}$ when we repeat this argument for every $U_{s j}$. Hence 3.4.s follows for all $s$.

3.5. Proof of The first half of TheOREM 1. Keep the same notations as 3.3 and 3.4.0. For each $i, j$ and $\alpha$ choose $V_{i j}^{\prime}$ small enough, then by (3.4.0.1), Lemma 3.1 and Argument 2.1 there exists an $\varepsilon$-approximation of $\tilde{f}_{i j \alpha P_{i j \alpha}} H_{i j}$ of order $r$ of the form $f_{i j \alpha}^{\prime} H_{i j}$, where $f_{i j \alpha}^{\prime}$ is a $C^{\omega}$ Nash function on $\mathbf{R}^{n}$ (to be precise, we have to construct $f_{i j \alpha}^{\prime}$ by downward induction on $i$ because $V_{i j}^{\prime}$ depends on $\left.U_{i j}-W_{i+1}\right)$. It also follows from (3.4.0.2) and Argument 2.1 that we have a $C^{\omega}$ Nash $\varepsilon$-approximation $g_{i j \alpha}$ of 
$f_{i j \alpha}^{\prime} H_{i j}$ of order $r$ of the form $f_{i j \alpha}^{\prime} G_{i j}$ for $G_{i j} \in N^{\omega}\left(\mathbf{R}^{n}\right)$. Hence $g_{i j \alpha}$ is a $2 \varepsilon$-approximation of $\tilde{f}_{i j \alpha P_{i j \alpha}} H_{i j}$ of order $r$. Put

$$
g=\sum_{\substack{|\alpha| \leqslant r \\ i, j}} g_{i j \alpha} h_{i}^{\alpha}
$$

Then by (3.4.0.3) and (3.4.0.4) we see

$$
\begin{aligned}
\left|D^{\beta}(f-g)\right| \leqslant & \sum_{i, j}\left|D^{\beta}\left\{f H_{i j}-\sum_{|\alpha| \leqslant r} g_{i j \alpha} h_{i}^{\alpha}\right\}\right| \\
\leqslant & \sum_{i, j}\left|D^{\beta}\left\{f H_{i j}-\sum_{|\alpha| \leqslant r} \tilde{f}_{i j \alpha P_{i j \alpha}} h_{i}^{\alpha} H_{i j}\right\}\right| \\
& +\sum_{\substack{|\alpha| \leqslant r \\
i, j}}\left|D^{\beta}\left\{\tilde{f}_{i j \alpha P_{i j \alpha}} h_{i}^{\alpha} H_{i j}-g_{i j \alpha} h_{i}^{\alpha}\right\}\right| \\
\leqslant & Q \varepsilon
\end{aligned}
$$

for all $\beta$ with $|\beta| \leqslant r$ and some polynomial $Q$ on $\mathbf{R}^{n}$ which does not depend on $\varepsilon$. Hence, diminishing $\varepsilon$ we can approximate $f$ by the $C^{\omega}$ Nash function $g$ in the $C^{r}$ topology. This completes the proof of the first half of Theorem 1.

3.6. Proof of THE LATTER Half of TheOREM 1. Let $i_{0}=\operatorname{codim} M_{3}$ in $\mathbf{R}^{n}$. By Corollary 5 in [9], we have $f_{0} \in N^{\omega}\left(\mathbf{R}^{n}\right)$ such that $f_{0}=f$ on $M_{3}$. Hence, considering $f-f_{0}$ in place of $f$, we can assume $f \equiv 0$ on $M_{3}$. Recall Corollary 6 in [9] which states that $M_{3}$ is $C^{\omega}$ Nash nonsingular, namely there exist $\theta_{1}, \ldots, \theta_{t} \in N^{\omega}\left(\mathbf{R}^{n}\right)$ such that $\theta_{1}^{-1}(0) \cap \cdots \cap \theta_{t}^{-1}(0)=M_{3}$ and $\operatorname{grad} \theta_{1}, \ldots, \operatorname{grad} \theta_{t}$ span the normal bundle of $M_{3}$ in $\mathbf{R}^{n}$. Put $\theta=\sum_{i=1}^{t} \theta_{i}^{2}$. Then we can add to 3.3 the following conditions: there exist, moreover, $\Lambda$ a subset of $\left\{(i, j): 0 \leqslant i \leqslant n, 1 \leqslant j \leqslant m_{i}\right\}, \bar{f}_{i j \alpha} \in N^{\omega}\left(U_{i j}\right)$ for all $(i, j) \notin \Lambda$ and $\alpha \in \mathbf{N}^{i}$ with $|\alpha| \leqslant r$, and minimal polynomials $\bar{P}_{i j \alpha}$ for $\bar{f}_{i j \alpha}$ such that

(3.3.6) $M_{3}=\bigcup_{(i, j) \in \Lambda} U_{i j}$;

(3.3.7) if $(i, j) \in \Lambda$ we can replace in (3.3.3) and (3.3.5) $i_{0}$ functions of $\left\{h_{i k}\right\}_{k=1, \ldots, i}$ by some $\theta_{k}$ 's and we use the notations $\left\{\bar{h}_{i j k}\right\}_{k=1, \ldots, i}$ for the new family, here we assume $\bar{h}_{i j 1}, \ldots, \bar{h}_{i j i_{0}}$ to be the replaced ones;

(3.3.8) $\left(\bar{f}_{i j \alpha}, \bar{P}_{i j \alpha}\right)$ have Property (A);

(3.3.9) for each $(i, j) \notin \Lambda, f / \theta-\sum_{|\alpha| \leqslant r} \tilde{\tilde{f}}_{i j \alpha \bar{P}_{i j}} h_{i}^{\alpha}$ is $r$-flat on $U_{i j}$.

As the proof of the above proceeds in the same way as 3.3 we omit it. We remark only that (3.3.7) implies that if we choose a local coordinate system at each point of $U_{i j} \subset M$ so that $\left\{\bar{h}_{i j k}\right\}_{k=1, \ldots, i}$ is its part, then $M_{3}$ and $U_{i j}$ become linear subspaces.

Recall $g$ in 3.5 which was the required approximation function. We modify $g$ as follows:

$$
\bar{g}=\sum_{\substack{(i, j) \notin \Lambda \\|\alpha| \leqslant r}} \bar{g}_{i j \alpha} h_{i}^{\alpha} \theta+\sum_{\substack{(i, j) \in \Lambda \\|\alpha| \leqslant r}} g_{i j \alpha} \bar{h}_{i j}^{\alpha},
$$

where $\bar{g}_{i j \alpha}$ are approximations of $\tilde{f}_{i j \alpha P_{i j \alpha}} H_{i j}$ defined in the same way as $g_{i j \alpha}$ in 3.5. It is obvious by definition that $\bar{g}$ is a $C^{\omega}$ Nash approximation of $f$ and the first part of $\bar{g}$ vanishes on $M_{3}$. Hence we need only

$$
\text { (3.6.1) } g_{i j \alpha} \equiv 0 \text { for }(i, j) \in \Lambda \text { and } \alpha_{1}=\cdots=\alpha_{i_{0}}=0 \text {. }
$$


For this it suffices by 3.5 that

(3.6.2) $\bar{P}_{i j \alpha}(z, x) \equiv z$ for the same $i, j$ and $\alpha$, which is equivalent to

(3.6.3) $\bar{f}_{i j \alpha} \equiv 0$ on $U_{i j}$ for the same $i, j$ and $\alpha$.

But this is clearly possible by the above remark and the method of proof of 3.3. Thus we have proved the latter half and, hence, Theorem 1.

REMARK 3.7. In Theorem 1 we do not need the compactness condition on $M_{3}$ if any $C^{\omega}$ Nash function on $M_{3}$ is extensible to $M_{1}$ and $M_{3}$ is " $C^{\omega}$ Nash nonsingular" in the sense in 3.6. The reason for this is clear by 3.6.

3.8. Proof of Corollary 2. Let $0 \leqslant r<\infty$ and $U_{3}$ be a semialgebraic set such that $\bar{U}_{1} \subset U_{3}$ and $\bar{U}_{3} \subset U_{2}$. Let $\varphi \in N^{r}\left(\mathbf{R}^{n}\right)$ such that $\varphi=1$ on $\bar{U}_{1}$ and $\varphi=0$ on $\mathbf{R}^{n}-U_{3}$, and apply Theorem 1 to $\varphi f \in N^{r}\left(\mathbf{R}^{n}\right)$. Then we obtain the required $g \in N^{\omega}\left(\mathbf{R}^{n}\right)$.

4. The approximation theorem for Nash manifolds. Let $0 \leqslant r \leqslant \omega$. A vector bundle $\xi=(E, p, B)$ is called a $C^{r}$ Nash vector bundle if the total space $E$ and the base space $B$ are $C^{r}$ Nash manifolds, $B$ is covered by a finite number of semialgebraic coordinate neighborhoods and all the coordinate functions and the projection $p$ are of class $C^{r}$ Nash. A $C^{r}$ Nash bundle map is naturally defined, and we call a $C^{r}$ Nash invertible bundle map a $C^{r}$ Nash isomorphism and two $C^{r}$ Nash vector bundles $C^{r}$ Nash isomorphic if there is a $C^{r}$ Nash isomorphism between them. Let $1 \leqslant k \leqslant n$ and $G_{n, k}$ denote the Grassmannian of $k$-linear subspaces in $\mathbf{R}^{n}$, put

$$
E_{n, k}=\left\{(\lambda, x) \in G_{n, k} \times \mathbf{R}^{n} \mid x \in \lambda\right\}
$$

and let $p_{G}: E_{n, k} \rightarrow G_{n, k}$ be the projection. Then the bundle $\xi_{G}=\left(E_{n, k}, p_{G}, G_{n, k}\right)$ naturally has a $C^{\omega}$ Nash vector bundle structure [8].

Proof of Theorem 3. Let $k$ denote the codimension of $M$ in $\mathbf{R}^{n}$. Let $\pi: M \rightarrow G_{n, k}$ denote the $C^{r-1}$ Nash map defined by $\pi(x)=$ the normal vector space of $M$ in $\mathbf{R}^{n}$ at $x, \pi^{\prime}$ a close $C^{\omega}$ Nash approximation of $\pi$ in the $C^{0}$ topology (Theorem 1), and $\pi^{* *} \xi_{G}=\left(\pi^{\prime *} E_{n, k}, p_{M}, M\right)$, the induced bundle of $\xi_{G}$ by $\pi^{\prime}$. Here we remark that $\pi^{\prime *} \xi_{G}$ is a Nash vector bundle of class $C^{r}$. Let us regard $M$ and $G_{n, k}$ as subsets of $\pi^{\prime *} E_{n, k}$ and $E_{n, k}$, respectively, through the zero cross-sections. Define a $C^{r}$ Nash $\operatorname{map} \varphi: \pi^{* *} E_{n, k} \rightarrow \mathbf{R}^{n}$ by

$$
\varphi(x, y, z)=x+z, \quad(x, y, z) \in \pi^{\prime *} E_{n, k} \subset M \times E_{n, k} \subset M \times G_{n, k} \times \mathbf{R}^{n} .
$$

Then we see easily (cf. the proof of Lemma 7 in [9]) that there exists a $C^{r} \mathrm{Nash}$ tubular neighborhood $V$ of $M$ in $\pi^{* *} E_{n, k}$ such that $\left.\varphi\right|_{V}$ is an imbedding. Put $W=\varphi(V)$ and $\psi=q \circ \varphi^{-1}: W \rightarrow E_{n, k}$, where $q: \pi^{\prime *} E_{n, k} \rightarrow E_{n, k}$ is the bundle map. Then $p_{M} \circ \varphi^{-1}: W \rightarrow M$ is a $C^{r}$ Nash tubular neighborhood of $M$ in $\mathbf{R}^{n}, \psi$ is transversal to $G_{n, k}$ and $\psi^{-1}\left(G_{n, k}\right)=M$. Apply Theorem 1 to $\psi$ and let $\psi^{\prime}: W \rightarrow E_{n, k}$ be a resultant $C^{\omega}$ Nash approximation in the $C^{r}$ topology such that $\psi^{\prime}$ is transversal to $G_{n, k}$. It is then easy to see that $M^{\prime}=\psi^{\prime-1}\left(G_{n, k}\right)$ is a $C^{\omega}$ Nash manifold, $\tau$ : $M \rightarrow M^{\prime}$ defined by $\tau(x)=\left(\left.p_{M} \circ \varphi^{-1}\right|_{W}\right)^{-1}(x) \cap M^{\prime}$ is a $C^{r}$ Nash diffeomorphism and, moreover, $\tau \rightarrow$ identity as $\psi^{\prime} \rightarrow \psi$ in the $C^{r}$ topology.

For the second part of the theorem, just use the latter half of Theorem 1. 
Proof of The last part of Theorem 3. By the first part we can assume $M$ is a $C^{\omega}$ Nash manifold in $\mathbf{R}^{n}$. Let $p: V \rightarrow M$ be a $C^{\omega}$ Nash tubular neighborhood in $\mathbf{R}^{n}$. Apply the first part to $M_{2} \subset \mathbf{R}^{n}$ Then we have a $C^{r}$ Nash imbedding $\pi: M_{2} \rightarrow \mathbf{R}^{n}$ arbitrarily close to the identity such that $\pi\left(M_{2}\right)$ is of class $C^{\omega}$ Nash. Moreover the above proof says that $\pi$ can be extended to a $C^{r}$ Nash diffeomorphism $\tilde{\pi}$ of $\mathbf{R}^{n}$. Hence choosing $\pi$ and, hence, $\tilde{\pi}$ close enough to the identity we obtain a required $C^{r}$ Nash imbedding $\left.p \circ \tilde{\pi}\right|_{M}$. Therefore Theorem 3 is proved.

Theorems 1 and 3 tell us the following.

Corollary 4.1. Let $1 \leqslant r<\infty$. Then the $C^{r}$ Nash diffeomorphism classes of all $C^{r}$ Nash manifolds is identical with the $C^{\omega}$ Nash diffeomorphism classes of all $C^{\omega}$ Nash manifolds.

REMARK 4.2. In spite of Corollary 4.1 there is a definite difference between the $C^{r}$ Nash category for $1 \leqslant r<\infty$ and the $C^{\omega}$ Nash category as shown in [11]. An abstract $C^{r}$ Nash manifold means a manifold with a finite system of coordinate neighborhoods of class $C^{r}$ Nash. To distinguish this we call a usual $C^{r} \mathrm{Nash}$ manifold affine. As [1,8 and 11] pointed out, an abstract $C^{\omega}$ Nash manifold is not necessarily affine. But if $0 \leqslant r<\infty$, then an abstract $C^{r}$ Nash manifold is always affine [11].

Proof of Corollary 4. We already know the corollary in the cases $r=\omega[9]$ and $r=0$ [12]. Hence by Corollary 4.1 it suffices to prove the following. Let $1 \leqslant r<\infty$ and let $M$ be a compact $C^{r}$ Nash manifold with boundary. Then

(1) $M$ is $C^{r}$ Nash diffeomorphic to some $C^{\omega}$ Nash manifold with boundary.

For the proof, consider the double $D M$ of $M$ with naturally defined abstract $C^{r}$ Nash manifold structure. Regard $M \subset D M$. Then, by Remark 4.2, $M \subset D M$ can be contained in some Euclidean space as $C^{r}$ Nash manifolds. Hence considering the pair ( $D M, \partial M)$ we obtain (1) by the last statement in Theorem 3.

THEOREM 4.3. Let $0 \leqslant r \leqslant \omega$. Let $\zeta=(E, p, M)$ be a $C^{0}$ vector bundle over a $C^{r}$ Nash manifold $M$. Then $\zeta$ has a unique $C^{r}$ Nash vector bundle structure up to a $C^{r}$ Nash isomorphism.

Proof. As the case of compact $M$ is easy, we assume $M$ is not compact. By Corollary 4 we regard $M$ as the interior of a compact $C^{r}$ Nash manifold $\tilde{M}$ with boundary. We also regard $\zeta$ as the induced bundle $f^{*} \xi_{G}$ of $\xi_{G}$ by some $C^{0}$ map $f$ : $M \rightarrow G_{n, k}$ for some $n$, where $k=\operatorname{dim} \zeta[13]$. We know [13] that if $f$ is homotopic to a $C^{0}$ map $g$, then $f^{*} \xi_{G}$ is equivalent to $g^{*} \xi_{G}$. So for the existence of $C^{r}$ Nash vector bundle structure on $\zeta$ we only need to find a $C^{r}$ Nash map $g: M \rightarrow G_{n, k}$ which is homotopic to $f$. Now $f$ is homotopic to the restriction to $M$ of a $C^{0} \operatorname{map} \tilde{f}: \tilde{M} \rightarrow G_{n, k}$ because $\tilde{M}$ has a collar. But by Theorem $1, \tilde{f}$ is approximated by a $C^{r}$ Nash map $\tilde{g}$ : $\tilde{M} \rightarrow G_{n, k}$. Hence choosing the approximation close enough we obtain the required $g=\left.\tilde{g}\right|_{M}$.

Proof of The Uniqueness. Assume $C^{r}$ Nash vector bundles $\zeta_{1}=\left(E_{1}, p_{1}, M\right)$, $\zeta_{2}=\left(E_{2}, p_{2}, M\right)$ and a bundle map $\Psi: \zeta_{1} \rightarrow \zeta_{2}$ of $C^{0}$ equivalence are given. Then we have to obtain a $C^{r}$ Nash isomorphism $\Phi: \zeta_{1} \rightarrow \zeta_{2}$. 
Put $E_{3}=\bigcup_{x \in M} L\left(p_{1}^{-1}(x), p_{2}^{-1}(x)\right)$ and $\zeta_{3}=\left(E_{3}, p_{3}, M\right)$, where $L($,$) means the$ space of linear maps and $p_{3}: E_{3} \rightarrow M$ is the projection. We want to give naturally $\zeta_{3}$ a $C^{r}$ Nash vector bundle structure. Let $\left\{U_{\alpha}\right\}_{\alpha \in A}$ and $\left\{V_{\beta}\right\}_{\beta \in B}$ be finite systems of semialgebraic coordinate neighborhoods of $\zeta_{1}$ and $\zeta_{2}$ respectively. Then for each $U_{\alpha}$ and $V_{\beta},\left(p_{3}^{-1}\left(U_{\alpha} \cap V_{\beta}\right), p_{3}, U_{\alpha} \cap V_{\beta}\right)$ has the trivial vector bundle structure. Hence the coordinate transformations of $\zeta_{1}$ and $\zeta_{2}$ give $\zeta_{3}$ an abstract $C^{r}$ Nash vector bundle structure in the sense of Remark 4.2, namely $E_{3}$ becomes an abstract $C^{r}$ Nash manifold. Therefore if $r<\infty, \zeta_{3}$ is a $C^{r}$ Nash vector bundle by Remark 4.2.

Consider the case $r=\omega$. Assume $E_{1}, E_{2} \subset \mathbf{R}^{n}$ and regard $M \subset E_{1}$ and $M \subset E_{2}$ through the zero cross-sections. For each $x \in M$ let $f_{1}(x)$ and $f_{2}(x)$ denote the tangent vector spaces of $p_{1}^{-1}(x)$ and $p_{2}^{-1}(x)$ at $x$ respectively. Then we see easily that $f_{1}, f_{2}: M \rightarrow G_{n, k}$ are $C^{\omega}$ Nash maps and that $f_{1}{ }^{*} \xi_{G}$ and $f_{2}{ }^{*} \xi_{G}$ are $C^{\omega}$ Nash isomorphic to $\zeta_{1}$ and $\zeta_{2}$ respectively. Hence we consider $f_{1}^{*} \xi_{G}$ and $f_{2}{ }^{*} \xi_{G}$ in place of $\zeta_{1}$ and $\zeta_{2}$. Put

$$
F=\left\{(\lambda, \mu, T) \in G_{n, k} \times G_{n, k} \times L\left(\mathbf{R}^{n}, \mathbf{R}^{n}\right): T \lambda \subset \mu, T \lambda^{\perp}=0\right\}
$$

and let $q: F \rightarrow G_{n, k} \times G_{n, k}$ be the projection. Then $\eta=\left(F, q, G_{n, k} \times G_{n, k}\right)$ is a $C^{\omega}$ Nash vector bundle (see the $C^{\omega}$ Nash manifold structure on $G_{n, k}$ in [8]), and $\left(f_{1}, f_{2}\right)^{*} \eta$ is $C^{\omega}$ Nash isomorphic to $\zeta_{3}$. Thus we have given $\zeta_{3}$ a $C^{r}$ Nash vector bundle structure in any case.

Let $E_{4}$ be the subset of $E_{3}$ consisting of linear isomorphisms and $p_{4}$ the restriction of $p_{3}$ to $E_{4}$. Then $E_{4}$ is an open semialgebraic subset of $E_{3}$ and $\zeta_{4}=\left(E_{4}, p_{4}, M\right)$ is a $C^{\omega}$ Nash fibre bundle with fibre $\operatorname{GL}(k, \mathbf{R})$. Now what we have to prove is that if $\zeta_{4}$ has a $C^{0}$ global cross-section $\psi$, then it has a $C^{r}$ Nash one $\varphi$.

Let us regard $M$ as contained in $\mathbf{R}^{n}$ so that $\bar{M}$ is compact and $\bar{M}-M$ consists of one point $a$ (see [9]). Then by [4] there exists a semialgebraic triangulation of $\bar{M}$ compatible with $\{a\} \cup\left\{U_{\alpha} \cap V_{\beta}\right\}_{\alpha \in A ; \beta \in B}$, i.e. a finite simplicial complex $K \subset \mathbf{R}^{n}$ and a semialgebraic homeomorphism $\tau:|K| \rightarrow \bar{M}$ such that $a$ or each $U_{\alpha} \cap V_{\beta}$ is the image of a union of some open simplices of $K$. Put $W_{\sigma}=\tau(\sigma)-a$ for $\sigma \in K$. Then $\left\{W_{\sigma}\right\}_{\sigma \in K}$ is a finite closed covering of $M$, moreover refining $\left\{U_{\alpha}\right\}$ and $\left\{V_{\beta}\right\}$ if necessary we can assume $\left.\zeta_{4}\right|_{W_{\sigma}}$ is $C^{r}$ Nash trivial for each $\sigma$.

Put

$$
\begin{aligned}
& K^{\prime}=\{\sigma \in K \mid \tau(\sigma) \ni a\}, \quad K^{\prime \prime}=K-K^{\prime}, \\
& M^{\prime}=\bigcup_{\sigma \in K^{\prime}} W_{\sigma} \text { and } M^{\prime \prime}=\bigcup_{\sigma \in K^{\prime \prime}} W_{\sigma}=\overline{M-M^{\prime}} .
\end{aligned}
$$

For the construction of $\varphi$, at first, we will define by induction a $C^{0} \mathrm{Nash}$ cross-section $\varphi^{\prime \prime}$ of $\left.\zeta_{4}\right|_{M^{\prime \prime}}$. If $\sigma \in K^{\prime \prime}$ is of dimension 0 we put $\varphi^{\prime \prime}\left(W_{\sigma}\right)=\psi\left(W_{\sigma}\right)$. So assume we have already defined $\varphi^{\prime \prime}$ on $\bigcup_{\operatorname{dim} \sigma<l} W_{\sigma}$ so that it is homotopic to the restriction of $\psi$ to $\bigcup_{\operatorname{dim} \sigma<l} W_{\alpha}$. Then for each $\sigma \in K^{\prime \prime}$ with $\operatorname{dim} \sigma=l$, the restriction of the homotopy to $\partial W_{\sigma}$ can be extended to $W_{\sigma}$ so that the extension of $\varphi^{\prime \prime}$ is of class $C^{0}$ Nash by the triviality of $\left.\zeta_{4}\right|_{W_{\sigma}}$, the Alexander trick and by Theorem 1 (see [12] for the Alexander trick). Hence we have globally $\varphi^{\prime \prime}$ on $M^{\prime \prime}$. Next extend $\varphi^{\prime \prime}$ to a $C^{0}$ Nash cross-section $\varphi_{0}$ of $\zeta_{4}$ by induction on $\operatorname{dim} \sigma$ for $\sigma \in K^{\prime}-\tau^{-1}(a)$ in the same way as above. Hence the case $r=0$ is proved. 
Assume $r>0$. For each $x \in M$ let $\rho_{x}$ denote the orthogonal projection of a semialgebraic tubular neighborhood of $p_{4}^{-1}(x)$ in $\mathbf{R}^{n}$. Put $P(x, y)=\rho_{x}(y)$. Then $P$ is a $C^{r-1}$ Nash map from an open semialgebraic set $X$ in $M \times \mathbf{R}^{n}$ to $E_{4}$. Furthermore, approximating $\rho_{x}$ by a $C^{r}$ map in the same way as the proof of Theorem 3 we can assume $P$ is of class $C^{r}$ Nash. Regard $\varphi_{0}$ as a map from $M$ to $E_{4}$ and apply Theorem 1 to $\varphi_{0}$. Then we have a $C^{r}$ Nash map $\chi: M \rightarrow E_{4}$ such that the graph of $\chi$ is contained in the domain of $P$, and, hence, $\varphi=P(x, \chi(x))$ is a required crosssection.

Problem. Let $M$ be a $C^{r}$ Nash manifold. We call a vector bundle over $M$ an abstract $C^{r}$ Nash vector bundle if the total space is an abstract $C^{r}$ Nash manifold and the same conditions on coordinates as a $C^{r}$ Nash vector bundle are satisfied. As pointed out in Remark 4.2, an abstract $C^{r}$ Nash vector bundle over $M$ is always affine if $r<\infty$. Is this the case for $r=\omega$ ?

ADDED IN PROOF. D. Pecker corrected the proofs of [2] in his thesis Fonction: approximation, extension, factorisation.

\section{REFERENCES}

1. D. R. Chillingworth and J. Hubbard, A note on nonrigid Nash structures, Bull. Amer. Math. Soc. 77 (1971), 429-431.

2. G. Efroymson, The extension theorem for Nash functions, Lecture Notes in Math., vol. 959, Springer-Verlag, Berlin and New York, 1982, pp. 343-357.

3. L. Hörmander, Linear partial differential operators, Springer-Verlag, Berlin and New York, 1969.

4. S. Lojasiewicz, Triangulations of semi-analytic sets, Ann. Scuola Norm. Sup. Pisa Cl. Sci. 18 (1964), 449-474.

5. __ Ensembles semi-analytiques, Inst. Hautes Études Sci., 1965.

6. T. Mostowski, Some properties of the ring of Nash functions, Ann. Scuola Norm. Sup. Pisa Cl. Sci. 3 (1976), no. 2, 245-266.

7. J. F. Nash, Real algebraic manifolds, Ann. of Math. (2) 56 (1952), 405-421.

8. R. Palais, Equivariant real algebraic differential topology, Part I, Smoothness categories and Nash manifolds, Notes, Brandeis Univ., 1972.

9. M. Shiota, Classification of Nash manifolds, Ann. Inst. Fourier (Grenoble) 33 (1983), 209-232.

10. _ Piecewise linearization of real analytic functions, Publ. Res. Inst. Math. Sci. Kyoto Univ. 20 (1984), 727-792.

11. __ Abstract Nash manifolds, Proc. Amer. Math. Soc. (to appear).

12. M. Shiota and M. Yokoi, Triangulations of subanalytic sets and locally subanalytic manifolds, Trans. Amer. Math. Soc. 286 (1984), 727-750.

13. N. Steenrod, The topology of fibre bundles, Princeton Univ. Press, Princeton, N. J., 1951.

14. B. Malgrange, Ideals of differentiable functions, Oxford Univ. Press, 1966.

Department of Mathematics, College of General Education, Nagoya University, Nagoya 464, JAPAN 